\title{
Spasticity in post-stroke patients: incidence and therapeutical approach
}

\author{
Ioana Stanescu ', Gabriela Dogaru ${ }^{2}$, Angelo Bulboaca' ${ }^{1}$, Dana Fodor', Adriana Bulboaca ${ }^{3}$
}

Corresponding author: : Gabriela Dogaru, E-mail address: dogarugabrielaumf@gmail.com

1. Department of Neurosciences, "Iuliu Hatieganu" University of Medicine and Pharmacy Cluj-Napoca, Clinical Rehabilitation Hospital 2.Department of Physical Medicine and Rehabilitation, "Iuliu Haţieganu" University of Medicine and Pharmacy Cluj-Napoca, Clinical

Rehabilitation Hospital

3. Department of Pathophysiology," Iuliu Haţieganu” University of Medicine and Pharmacy, Cluj-Napoca, Clinical Rehabilitation Hospital

\begin{abstract}
Stroke is a leading cause of serious long-term disability: about one third of patients developing spasticity of the affected limbs. Spasticity is characterized by a velocity-dependent increase in resistance to passive movement, and is one of the "positive signs" of upper motor neuron syndrome. Spasticity induces pain, ankylosis, tendon retraction, increasing motor deficit, which may limit the efficacy of rehabilitation methods . Spasticity is also correlated with activity limitations, and reduces quality of life of patients and caregivers. Assessment of post-stroke spasticity requires first clinical examination; scales as Ashworth Modifyed scale and Tardieu Modifyed scale are useful quantitative tools. Treatment of spasticity is often challenging for the rehabilitation team, requiring a multidisciplinary approach. Therapeutic interventions include physical therapy, occupational therapy, use of assistive devices, pharmacological treatment and injectable treatment. Botulinum toxin injections in spastic upper and lower limb muscles have significant effect in reducing muscle tone and improving passive function in affected limbs, and should also be considered for improvement of active function.
\end{abstract}

Key words: spasticity, stroke, rehabilitation, botulinum toxin,

\section{Introduction}

Stroke is a disabilitating disease. According to the Heart Disease and Stroke Statistics - 2018 Update [1], stroke prevalence increases with advancing age in both males and females, affecting $2.7 \%$ of persons in the United States. Stroke is a leading cause of serious long-term disability: about half of stroke survivors remain with some degree of physical or cognitive impairment, and $3 \%$ of males and $2 \%$ of females in general population reporting post-stroke disability, females often having greater disability [1].

More than two thirds of stroke survivors receive rehabilitation services after hospitalization. In data from 2011, 19\% of Medicare patients were discharged to inpatient rehabilitation facilities, 25\% were discharged to skilled nursing facilities, and $12 \%$ received home health care [2].

Spasticity was first described by Lance, as a state of increased muscle tone with exaggerated reflexes characterized by a velocity-dependent increase in resistance to passive movement [3]. It is a "positive sign", component of the upper motor neuron syndrome, together with extensor of flexor spasms, spastic dystonia, clonus, spastic co-contraction, and exagerated deep tendon reflexes. All these abnormal features will lead to abnormal limb positioning.

The prevalence of spasticity in stroke patients in any limb is in the range of $25 \%$ to $43 \%$ in the first year after stroke [4]. On the basis of its time course, poststroke spasticity could be divided in acute (less than one month after stroke), sub-acute (between one month and six months after stroke onset), and chronic (occurring more than six months) [5]. Early spasticity appears in 4 to $27 \%$ of patients during the first 6 weeks after stroke onset [6]. In the first 3 months, the incidence of upper limb spasticity is $33 \%$ and increases with time [7].

Patients with strokes located in the basal ganglia and internal capsule had the highest spasticity incidence [7].

Spasticity distribution primarily affects the elbow (79\% of patients), the wrist $(66 \%)$ and the ankle (66\%) [8]. Most common pattern of upper limb poststroke spasticity is internal rotation and adduction of the shoulder coupled with flexion at the elbow, the wrist and the fingers. In the lower limbs, adduction and extension of the knee with equinovarus foot is the most observed pattern [9], [10].

Predictors of spasticity development are severe proximal and distal limb weakness early after stroke, low Barthel Index, sensory deficit, lesion volume, stroke location and associated diseases [6]. Early identification of these factors is essential for treatment decision, which will minimise long-term complications and will provide better motor and functional outcomes in stroke patients [6]. 
Patients with acute and subacute stroke with severe motor and sensorimotor deficits required close monitoring by stroke and rehabilitation physicians and by other members of the rehabilitation team, in order to detect spasticity.

Spasticity could induce pain, ankylosis, tendon retraction, and increase muscle weakness in patients, which may limit the efficacy of rehabilitation methods [11]. Spasticity is correlated with activity limitations of daily function, and reduces quality of life of patients and caregivers [4].

Spasticity is induced by the dissociation or disintegration of motor responses from sensory input, leading to hyperexcitability of the segmental central nervous system [12].

Assessment of post-stroke spasticity requires first clinical evaluation: inspection of limb posture, examination of passive and active joint range of motion, voluntary muscle contraction in affected limb, deep tendon reflexes and sensory examination. Scales are developed and validated to assess the degree and angle of muscle contraction [5]. The two most commonly used scales are Modified Ashworth Scale and Modified Tardieu Scale [11].

The Modified Ashworth Scale (MAS) measures the level of resistance to passive movement [13]. Its quotations are (after Bohannon):

$0=$ No increase in muscle tone

$1=$ Slight increase in muscle tone, manifested by a catch or by minimal resistance at the end of the range of motion (ROM) when the affected part(s) is (are) moved in flexion or extension

$1+=$ Slight increase in muscle tone, manifested by a catch, followed by minimal resistance throughout the remainder (less than half) of the ROM

$2=$ More marked increase in muscle tone through most of the ROM, but affected part(s) easily moved

3 = Considerable increase in muscle tone, passive movement difficult

$4=$ Affected part(s) rigid in flexion or extension

The Modified Tardieu Scale (MTS) measures the velocity of passive joint movement and the angle of contraction [14], [15]. Its items are:

$\mathrm{X}$ : Quality of movement mobilization

$0=$ No resistance throughout the course of the passive movement

$1=$ Slight resistance throughout the course of passive movement, no clear catch at a precise angle

$2=$ Clear catch at a precise angle, interrupting the passive movement, followed by release
$3=$ Fatigable clonus with less than 10 seconds when maintaining the pressure and appearing at the precise angle

4 = Unfatigable clonus with more than 10 seconds when maintaining the pressure and appearing at a precise angle

$5=$ Joint is fixed

$\mathrm{V}$ : Measurements take place at three different velocities

$\mathrm{V} 1$ - as slow as possible

V2 - Speed of limb segment falling under gravity

V3 - As fast as possible

$\mathrm{Y}$ : Angle of catching (muscle reaction)

Classification of spasticity depends on the scores obtained on Ashworth scale: mild spasticity is considered at 1 point, moderate at 2 points and severe if the Ashworth score is 3 [16]. .

Other functional parameters that needs to be tested in spastic post-stroke patients are active upper limb function (scales - Action Research Arm Test, Modified Frenchay Scale)[17] and the Disability Assessment Scale, which assesses hygiene, dressing, limb position and pain [18].

Treatment of spasticity is often challenging for the rehabilitation team, requiring a multidisciplinary approach. Therapeutic interventions include physical therapy, occupational therapy, use of assistive devices, pharmacological treatment, and in selected cases surgical treatment.

Role of physical therapy in ameliorating spasticity is well known. Evidences support a positive effect of muscle stretching and muscle reinforcement on spasticity [19]. Muscle stretching can be apply by moving the joint manually or by mechanical devices; includes active stretching, passive stretching, prolonged positioning, isotonic and isokinetic stretching. The goal is to increase the extensibility of soft tissues, normalize muscle tone and to reduce contracture-related pain. Muscle strengthening could be obtained by muscle training and biofeedback procedures [19]. The efficacy of physical agents is also reported: shock wave therapy, ultrasound therapy, cryotherapy, thermotherapy, hydrotherapy and vibratory stimulation have been used in poststroke spasticity. However, only vibration treatment applied to spastic muscle groups might be considered, but did not have long-term effects, according to current Guidelines for adult stroke rehabilitation and recovery [4]. 
Electrical stimulationused TENS (Transcutaneous Electrical Nerve Stimulation) - with controversial effect and NMES (NeuroMuscular Electrical Stimulation), which may be reasonable to improve spasticity temporarily (in combination to other nonpharmacological therapies), but is ineffective in improving functional hand use [4], [11],[19].

Role of assistive devices is also controversial in poststroke spasticity. Splints or ortheses, which can be placed and left for several hours, were used to obtain reductions in spasticity and pain, improvement of function, and prevention of contracture and deformity [11]. The use of resting hand splints is not effective for preventing muscle contracture of paretic upper limb early after stroke and also in reducing wrist and finger spasticity [4]. Casting immobilizes the limb in a stretch position, inducing prolonged muscle stretching. Short duration ankle casting may be used to facilitate reduction of plantarflexor spasticity (but after botulinum toxin treatment), but . taping has no effect in these situations [4].

Oral antispastic agents aim to reduce muscle tone, acting on the CNS (central nervous system) or directly on the muscle. Traditional mucle relaxants should be introduced when the patient suffers from motor disability due to spasticity, but have a controversial efficacy [11]. They have a limited effect on reducing general spasticity and dystonia, but they have dose-limiting side effects (weakness, tiredness and sedation) [4], [17]. Agents frequently used are : baclofen (5-20 mg 3-4 times daily), clonazepam (0.5-1.0 mg once daily - at bedtime), diazepam (5$20 \mathrm{mg} 3$ times daily), gabapentin (240-360 mg daily), tizanidine (4-36 $\mathrm{mg}$ daily) and dantrolene (25-100 mg 4 times daily) [11]

Phenol or alcohol injections are no more used because of severe local adverse reactions [11]. Intrathecal baclofen therapy (pump) is effective in reducing generalized spasticity in stroke patients which do not respond well to other interventions or experienced severe adverse effects from other treatments [4].

Injection of botulinum toxin (BoT) in spastic mucles have shown efficacy in reducing spasticity. Improvements were attributable to the lowered resistance to muscle stretch during passive movements. Mechanism of action of BoT on spastic muscle involved reduction in spastic co-contraction (inappropriate antagonistic co-activation during volitional command on an agonist), decreasing of spastic dystonia, stretch facilitation and lengthening of the injected muscle [20].

Current guidelines recommend botulinum toxin for reducing muscle tone and improving passive function in adults with spasticity; BoT should also be considered for improvement of active function.

BoT is the most potent neurotoxin; it is produced by Clostridium botulinum, and induces a blockade of neuromuscular transmission, with a paralytic effect on muscles. BoT is available in 2 serotypes: A and B. There are 4 commercial approved preparations: onabotulinumtoxinA (Botox), abobotulinumtoxinA (Dysport), incobotulinumtoxinA (Xeomin) and rima botulinumtoxinB (Myobloc). There are important pharmacological differences between BoT preparations, including latency, duration and intensity of the effect [21].

The effects of botulinum toxin injections are generally dose dependent, are realized within 3 to 7 days following injection, and last approximately 2 to 4 months [22].

Doses used in treatment are variable, ranging from 75 to a maximum of 500 units of onabotulinumtoxin and 500 to 1500 units of abobotulinumtoxinA [17]. Generally all doses of botulinum toxin resulted in reduction in muscle tone; however, increasingly higher doses were associated with greater muscle weakening [22].

For upper limb post-stroke spasticity, 2010 American Academy of Neurology guidelines [21] stated that aboBoT-A (Botox), onaBoT-A (Dysport) and incoBoT-A (Xeomin) were safe and demonstrated significant reductions in upper limb muscle tone (measured by the MAS) and improvements in passive arm function.

In spastic lower limb, the most disabling problem is spastic equinovarus foot, frequent complication following stroke, caused by increased tone in a pattern that includes spasticity of the gastrocnemius and tibialis posterior muscles [22]. Injections with aboBoT-A (Botox) and onaBoT-A (Dysport) in the gastrocnemius and soleus muscles are safe and effective for the reduction of lower limb spasticity [4], [21].

However, data are inadequate to support the efficacy of BoT in improvement of active function of spastic upper limb or lower limb [21]. There is no evidence to sustain an effect of BoT injections on functional upper limb use, although it improves upper limb positioning for daily activities (dressing, hygiene) [4]. 
Another study showed that BoT injections in spastic upper limb have also a small, but significant effect on improving daily activities, as measured by Disability Assessment Scale (DAS), a measure of self-reported disability [4].

For active lower limb function, BoT injections in the ankle plantarflexor and invertor muscles reduce spasticity, indirectly improving gait speed [4]. BoT injections in rectus femoris muscle will also improve knee extension during walking [4].

The maximal effect of the BoT treatment was observed at 4 weeks after injection, and the improvement in MAS scores were sustained up to 12 weeks [17].

Timing of BoT injections was also assessed. There are evidences supporting early administration of abobotulinumtoxinA in the first 2 to 12 weeks after stroke. This will not only improve spasticity (as measured by MAS), but the improvement lasts longer, increasing significantly the time until reinjection, compared with placebo. The hypothesis is that early BoT injections could induce a delay in symptomatic spasticity development, and suggest a prolonged effect of BoT if administrated early [23]. Despite beneficial effect of BoT injections in decreasing spasticity, there are concerns about the relatively high cost of treatment and about the smaller or absent effect on active function [22]. For this reason, some studies focused on the impact of reducing spasticity by BoT treatment on functional disability and quality of life. BoT treatment improves impression of functional disability assessed by the Global Assessment Scale (which consider also patient's perspective), at 4 and 6 weeks after treatment [17], but the effect did not last after week 12.

Botulinum toxin injections have also demonstrated significant greater global benefit, but no significant difference in quality of life (QOL) of stroke patients. In a study performed by McCrory et al, which used 2 injections of BoT in the distal spastic upper limb at 12 weeks interval, no significant benefit in improving quality of life was demonstrated using the AQoL (Assessment of Quality of Life) scale [24].

The possible explanation for lack of effect of BoT treatment on patient's quality of life is that BoT treatment should not be seen as a treatment in isolation, but as part of a more comprehensive treatment strategy that also involves physiotherapy, bracing, balneotherapy with the use of natural therapeutic factors and pharmacotherapy [25].

Surgical treatment of spasticity is mainly used for severe cases or for the effects induced by spasticity that become functional impairments (e.g. irreductible equinus) [11].

In conclusion, spasticity is a disabling post-stroke condition, which requires treatment. The evident diagnosis is followed by challenging management strategies. Spasticity treatment needs a multidisciplinary approach, in which botulinum toxin focal injections have a central role, targeting increase in muscle tone; other treatment modalities, such as oral pharmacological agents, rehabilitative interventions and pain management, aimed to improve functional disability of the patients.

\section{References}

1. Benjamin EJ, Virani SS, Callaway CW, Chamberlain AM, Chang AR, Cheng S, Chiuve SE, Cushman M, Delling FN, Deo R, de Ferranti SD, Ferguson JF, Fornage M, Gillespie C, Isasi CR, Jiménez MC, Jordan LC, Judd SE, Lackland D, Lichtman JH, Lisabeth L, Liu S, Longenecker CT, Lutsey PL, Mackey JS, Matchar DB, Matsushita K, Mussolino ME, Nasir K, O'Flaherty M, Palaniappan LP, Pandey A, Pandey DK, Reeves MJ, Ritchey MD, Rodriguez CJ, Roth GA, Rosamond WD, Sampson UKA, Satou GM, Shah SH, Spartano NL, Tirschwell DL, Tsao CW, Voeks JH, Willey JZ, Wilkins JT, Wu JH, Alger HM, Wong SS, Muntner P; American Heart Association Council on Epidemiology and Prevention Statistics Committee and Stroke Statistics Subcommittee. Heart Disease and Stroke Statistics2018 Update: A Report From the American Heart Association. Circulation. 2018 Mar 20;137(12):e67e492

2. Buntin MB, Colla CH, Deb P, Sood N, Escarce JJ. Medicare spending and outcomes after postacute care for stroke and hip fracture. Med Care.2010;48:776784.

3. Lance JW. The control of muscle tone, reflexes and movement: Robert Wartenberg lecture. Neurology. 1980;30:1303-1313.

4. Winstein CJ, Stein J, Arena R, Bates B, Cherney LR, Cramer SC, Deruyter F, Eng JJ, Fisher B, Harvey RL, Lang CE, MacKay-Lyons M, Ottenbacher KJ, Pugh S, Reeves MJ, Richards LG, Stiers W, Zorowitz RD; American Heart Association Stroke Council, Council on Cardiovascular and Stroke Nursing, Council on Clinical Cardiology, and Council on Quality of Care and Outcomes Research. Guidelines for Adult Stroke Rehabilitation and Recovery. A Guideline for 
Healthcare Professionals From the American Heart Association/ American Stroke Association. Stroke. 2016 Jun;47(6):e98-e169.

5. Sandrini G, Baricich A, Cisari C, Paolucci S, Smania N, Picelli A.

6. Management of spasticity with onabotulinumtoxinA: practical guidance based on the italian real-life poststroke spasticity survey. Funct Neurol. 2018 Jan/Mar;33(1):37-43

7. Wissel J, Verrier M, Simpson DM, Charles D, Guinto P, Papapetropoulos S, Sunnerhagen KS. Post-stroke spasticity: predictors of early development and considerations for therapeutic intervention. PM\&R. 2015 Jan;7(1):60-7.

8. Jin Y, Zhao Y. Post-stroke Upper Limb Spasticity Incidence for Different Cerebral Infarction Site. Open Med (Wars). 2018 Jun 1;13:227-231.

9. Wissel J, Schelosky LD, Scott J, Christe W, Faiss JH, Mueller J. Early development of spasticity following stroke: A prospective, observational trial. Journal of Neurology 2010;257:1067-1072.

10. Hefter H, Jost WH, Reissig A, Zakine B, Bakheit AM, Wissel J. Classification of posture in poststroke upper limb spasticity: A potential decision tool for botulinum toxin A treatment? International Journal of Rehabilitation Research 2012;35:227-233

11. Marciniak C. Poststroke hypertonicity: Upper limb assessment and treatment. Topics in Stroke Rehabilitation 2011;18:179-194.

12. Thibaut A, Chatelle C, Ziegler E, Bruno MA, Laureys S, Gosseries O. Spasticity after stroke: Physiology, assessment and treatment. Brain Inj. 2013;27(10):1093-105.

13. Mayer NH, Esquenazi A. Muscle overactivity and movement dysfunction in the upper motoneuron syndrome. Physical Medicine \& Rehabilitation Clinics of North America 2003;14:855-883.

14. Bohannon R, Smith M. Inter rater reliability of a modified Ashworth Scale of muscle spasticity. Physical Therapy 1987;67: 206-207.

15. Tardieu G, Shentoub S, Delarue R. A la recherche d'une technique de mesure de la spasticite. Revista de Neurologia 1954;91:143-144.

16. Held J, Pierrot-Deseilligny E. Reeducation Motrice des Affections Neurologiques. Paris: J.B. Bailleres; 1969.

17. Kong KH, Lee J, Chua S. Occurrence and temporal evolution of upper limb spasticity in stroke patients admitted to a rehabilitation unit. Arch Phys Med Rehabil. 2012 Jan; 93(1):143-8.

18. Dong $\mathrm{Y}, \mathrm{Wu} \mathrm{T}, \mathrm{Hu} \mathrm{X}$, Wang T. Efficacy and safety of botulinum toxin type A for upper limb spasticity after stroke or traumatic brain injury : a systematic review with meta-analysis and trial sequential analysis. European Journal of Physical and Rehabilitation Medicine 2017 April;53(2):256-67.

19. Brashear A, Zafonte R, Corcoran M, Galvez-Jimenez N, Gracies JM, Gordon MF, McAfee A, Ruffing K, Thompson B, Williams M, Lee CH, Turkel C. Interand intrarater reliability of the Ashworth Scale and the Disability Assessment Scale in patients with upper limb poststroke spasticity. Archives of Physical Medicine \& Rehabilitation 2002;83:1349-1354.

20. Smania N, Picelli A, Munari D, Geroin C, Ianes P, Waldner A, Gandolfi M. Rehabilitation procedures in the management of spasticity. Eur J Phys Rehabil Med. 2010 Sep;46(3):423-38.

21. Simpson DM, Gracies JM, Graham HK, Miyasaki JM, Naumann M, Russman B, Simpson LL, So Y; Therapeutics and Technology Assessment Subcommittee of the American Academy of Neurology. Assessment: Botulinum neurotoxin for the treatment of spasticity (an evidence-based review): report of the Therapeutics and Technology Assessment Subcommittee of the American Academy of Neurology. Neurology. 2008 May 6;70(19):1691-8.

22. Simpson DM, Hallett M, Ashman EJ, Comella CL, Green MW, Gronseth GS, Armstrong MJ, Gloss D, Potrebic S, Jankovic J, Karp BP, Naumann M, So YT, Yablon SA. Practice guideline update summary: Botulinum neurotoxin for the treatment of blepharospasm, cervical dystonia, adult spasticity, and headache: Report of the Guideline Development Subcommittee of the American Academy of Neurology. Neurology. 2016 May 10;86(19):181826.

23. Teasell R, Foley N, Pereira S, Sequeira K, Miller T. Evidence to practice: botulinum toxin in the treatment of spasticity post stroke. Top Stroke Rehabil. 2012 Mar-Apr;19(2):115-21

24. Rosales RL, Balcaitiene J, Berard H, Maisonobe P, Goh KJ, Kumthornthip W, Mazlan M, Latif LA, Delos Santos MMD, Chotiyarnwong C, Tanvijit P, Nuez O, Kong KH. Early AbobotulinumtoxinA (Dysport) in Post-Stroke Adult Upper Limb Spasticity: ONTIME Pilot Study. Toxins (Basel). 2018 Jun 21;10(7).

25. McCrory $\mathrm{P}$, Turner-Stokes L, Baguley IJ, et al. Botulinum toxin A for treatment of upper limb spasticity following stroke: a multi-centre randomized placebo-controlled study of the effects on quality of life and other person-centred outcomes. J Rehabil Med 2009;41:536-544.

26. Dogaru G, Ispas A, Bulboacă A, Motricală M, Stănescu I. Influence of balnear therapy at Băile Tuşnad on quality of life of post-stroke patients. Balneo Research Journal. 2017;8(4):201-20 